\title{
Down with the Big Bang
}

Apart from being philosophically unacceptable, the Big Bang is an over-simple view of how the Universe began, and it is unlikely to survive the decade ahead.

BY now, of course, every schoolboy knows that the quintessential point in space-time is the occasion of the Big Bang with which the Universe is supposed to have begun. That, the argument goes, is when all the matter now in the Universe was concentrated in an infinitesimal volume so that, among other things, there could have been no ambiguity about the time such as arises in the present Universe, in which the age of distant galaxies must be less than that of ours. At the Big Bang, the Universe, whatever its constituents, must have been a point-like space at a well-defined instant.

In all respects save that of convenience, this view of the origin of the Universe is thoroughly unsatisfactory. For one thing, the implication is that there was an instant at which time literally began and, so, by extension, an instant before which there was no time. That in turn implies that even if the origin of the Universe may be successfully supposed to lie in the Big Bang, the origin of the Big Bang itself is not susceptible to discussion.

It is an effect whose cause cannot be identified or even discussed. Even the notion that the present appearance of the Universe may represent but one cycle in the oscillation of a Universe whose total mass is enough to hold it together gravitationally (which seems unlikely) would not resolve the philosophical difficulty that an important issue, that of the ultimate origin of our world, cannot be discussed.

Creationists and those of similar persuasions seeking support for their opinions have ample justification in the doctrine of the Big Bang. That, they might say, is when (and how) the Universe was created. The reality of the event is accepted. The question of its cause, in the absence of time, is a matter for the imagination. Moderate creationists are no doubt content with that inference.

Luckily for the rest of us, moderate creationists' more impatient (and noisy) brethren seem more concerned to demonstrate that the whole world began just a few thousand years ago, which is why they have impaled themselves on the hook of trying to disprove the relatively recent (and terrestrial) geological record. But, in the long run, the impatient creationists will have to retreat to the Big Bang, for which reason it may be important that Donald Lynden-Bell, J. Katz and J.H. Redmount (the first and last from the University of Cambridge's Institute of
Astronomy) have just published a calculation showing that, unless the average density of the Universe is so great that the present expansionist phase will be halted, the Big Bang could not have been a single point in space-time, but must at the very least have been a line therein (Mon. Not. R. astr. Soc. 239, 201; 1989).

The argument is intricate in its detail but simple in its essence: if you seek an intuitively simple description of the real Universe, you might profitably begin by insisting on the right to use intuitive (but, in reality, mistaken) notions of what space and time are like - flat and uniform respectively.

That leads you to think of embedding the real Universe in a geometrical framework with at least one more dimension. Intuitive rules of geometry correspond to Minkowski space-time, which is flat and uniform, but which has the disadvantage of allowing only for universes that are empty of matter. In the real world, which is not empty, space-time is curved in a manner determined by the large-scale distribution of matter (Mach's principle), but that Universe can be made more tractable by embedding it in a Minkowski space-time with an extra dimension.

That means that intuitive concepts of what the world is like can be applied to a discussion of how the matter-filled Universe behaves. Lynden-Bell and his associates show that their trick works well for a two-dimensional universe (a sheet of matter) embedded in three-dimensional flat space. If the density of the matter in the sheet is great enough, it will first expand as a disk, and then contract (onto the 'Big Crunch'). They go on to show that when geometrically three-dimensional universes are embedded in flat space-time with an extra dimension, it is necessary to reconstruct the beginning of the Universe by cutting a section through the space with an extra dimension. The result is not a unique point, but a line. What that implies is that the impression that the Universe began with a Big Bang would be no less (and no more) valid from elsewhere (in time as well as space) in our own Universe, but that the estimates of when the Big Bang happened would usually be discordant with each other. On a more distant galaxy, your qualitative appreciation of the course of events through successive generations of particles might be very different from that now current here.

Purists will no doubt find room to protest that the argument as outlined here is insubstantial. By what right can one require of the real world that it should be describable in language that is intuitively simple, obeying the prejudices of flat space-time, for example? Because that is how we think. Are not Newton's laws simply a means of codifying our knowledge of how the behaviour of the real world differs from the expectations of our flat and uniform intuition? That is merely another way of drawing attention to one of the more flagrant gaps in the epistemology of our understanding of the world we live in.

Ernst Mach was Einstein's original intellectual hero because he originated the positivist view that has been the sheetanchor of observational science for most of the past century: reality is what you can measure, and what you cannot measure does not count. This more general principle is just as valid now as it was a century ago, as can be told from its utility in the interpretation of quantum mechanics, for example. But is it possible that the time has come for a closer enquiry into what exactly is to be understood by quantities counted as observables?

In cosmology, Doppler shifts are measurable, velocities can be inferred and, given a suitable model of the Universe, so can distances. But is it possible to measure the curvature of the Universe which, in what seems to be largely a flat space like regime, is linked primarily with newtonian gravitation? Or, in particle physics, if the properties of successive generations of material objects such as atoms, nucleons and quarks should seem related to each other like the objects in a stack of Russian dolls, is there some more durable observable whose measurement would be more illuminating?

Luckily, at least where the structure of the Universe is concerned, there may not be long to wait for an answer. It is unthinkable that the launch of the Hubble Space Telescope can be long delayed, and it is exceedingly improbable that the succeeding decade will allow the persistence of present views of how the Universe is constructed. The Big Bang itself is the pinnacle of a chain of inference which provides no explanation at present for quasars and the source of the known hidden mass in the Universe. It will be a surprise if it somehow survives the Hubble telescope.

John Maddox 